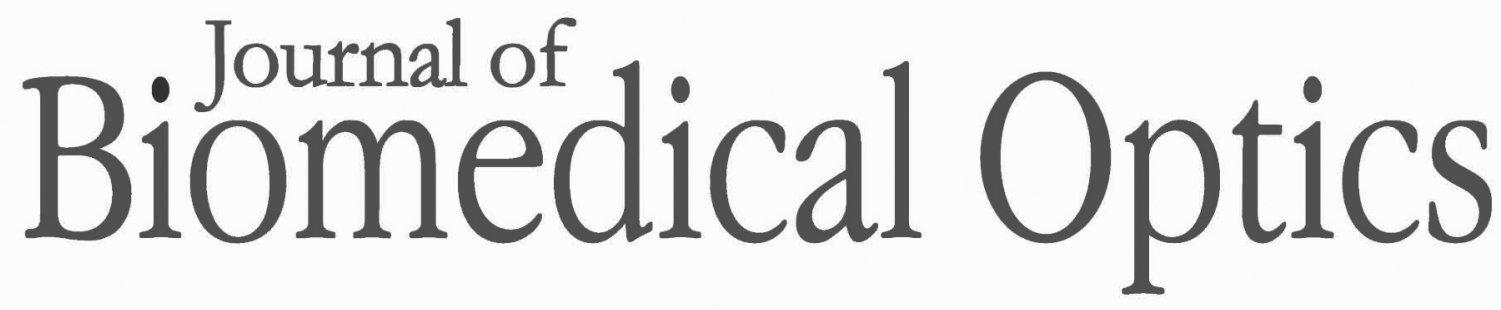

\title{
Multispectral Mueller polarimetric imaging detecting residual cancer and cancer regression after neoadjuvant treatment for colorectal carcinomas
}

\author{
Angelo Pierangelo \\ Sandeep Manhas \\ Abdelali Benali \\ Clément Fallet \\ Jean-Laurent Totobenazara \\ Maria-Rosaria Antonelli \\ Tatiana Novikova \\ Brice Gayet \\ Antonello De Martino \\ Pierre Validire
}




\title{
Multispectral Mueller polarimetric imaging detecting residual cancer and cancer regression after neoadjuvant treatment for colorectal carcinomas
}

\author{
Angelo Pierangelo, ${ }^{a}$ Sandeep Manhas, ${ }^{a}$ Abdelali Benali, ${ }^{b}$ Clément Fallet, ${ }^{\text {a }}$ Jean-Laurent Totobenazara, ${ }^{\text {a }}$ Maria-Rosaria \\ Antonelli, ${ }^{a}$ Tatiana Novikova, ${ }^{a}$ Brice Gayet, $^{c}$ Antonello De Martino, ${ }^{a}$ and Pierre Validire ${ }^{b}$ \\ aLaboratoire de Physique des Interfaces et Couches Minces, Ecole Polytechnique, Centre National de la Recherche Scientifique, Palaiseau 91128, \\ France \\ bInstitut Mutualiste Montsouris, Département d'Anatomopathologie, 42 Boulevard Jourdan 75014, Paris, France \\ 'Institut Mutualiste Montsouris, Département médico-chirurgical de pathologie digestive, 42 Boulevard Jourdan 75014, Paris, France
}

\begin{abstract}
This work is devoted to a first exploration of Mueller polarimetric imaging for the detection of residual cancer after neoadjuvant treatment for the rectum. Three samples of colorectal carcinomas treated by radiochemotherapy together with one untreated sample are analyzed ex vivo before fixation in formalin by using a multispectral Mueller polarimetric imaging system operated from 500 to $700 \mathrm{~nm}$. The Mueller images, analyzed using the Lu-Chipmann decomposition, show negligible diattenuation and retardation. The nonirradiated rectum exhibits a variation of depolarization with cancer evolution stage. At all wavelengths on irradiated samples, the contrast between the footprint of the initial tumor and surrounding healthy tissue is found to be much smaller for complete tumor regression than when a residual tumor is present, even at volume fractions of the order of $5 \%$. This high sensitivity is attributed to the modification of stromal collagen induced by the cancer. The depolarization contrast between treated cancer and healthy tissue is found to increase monotonously with the volume fraction of residual cancer in the red part of the spectrum. Polarimetric imaging is a promising technique for detecting short-time small residual cancers, which is valuable information for pathological diagnosis and patient management by clinicians. (C) The Authors. Published by SPIE under a Creative Commons Attribution 3.0 Unported License. Distribution or reproduction of this work in whole or in part requires full attribution of the original publication, including its DOI. [DOI: 10.1117/1.JBO.18.4.046014]
\end{abstract}

Keywords: multispectral Mueller polarimetry; Mueller imaging; neoadjuvant treatment for rectum; residual cancer detection; cancer detection and staging.

Paper 12763R received Nov. 28, 2012; revised manuscript received Feb. 20, 2013; accepted for publication Feb. 21, 2013; published online Apr. 23, 2013.

\section{Introduction}

Gastrointestinal malignancies are among the most important causes of cancer-related deaths. Colorectal cancer is one of most commonly diagnosed cancer in the world. ${ }^{1}$ More than $90 \%$ of primitive colorectal malignant tumors are adenocarcinomas, originating from glandular colorectal epithelium. Currently surgery is the most radical treatment of cancer with a curative purpose, provided that the disease is detected at early stage and its size is limited.

Locally advanced colorectal adenocarcinomas (corresponding with Liberkühnian adenocarcinomas) diagnosed before surgery as T3 to T4 lesions, with or without regional metastatic lymph nodes, benefit from neoadjuvant treatment combining radio- and/or chemotherapy, followed by total excision of mesorectum. $^{2-5}$ Application of neoadjuvant treatments before surgery reduces the probability of local relapse and makes surgery easier. ${ }^{6}$ After surgery, the role of pathologist is crucial to choose the appropriate medical treatment to increase patient survival. Pathologists are involved in the macroscopic and microscopic analysis of resected surgery specimens ${ }^{7}$ for the ypTNM staging (where y stands for neoadjuvant therapy,

Address all correspondence to: Angelo Pierangelo, Laboratoire de Physique des Interfaces et Couches Minces, Ecole Polytechnique, Centre National de la Recherche Scientifique, Palaiseau 91128, France. Tel: +33169334364; E-mail: angelo.pierangelo@poly.polytechnique.fr $\mathrm{p}$ for pathology and TNM is the usual score for cancers, with T0 to T4 for the initial tumor, N0 to N2 for lymph nodes and M0 or M1 for metastases ${ }^{8}$ ), the determination of the patient sensitivity to the treatment, the evaluation of the circumferential margin $^{9}$ and of the tumor regression. ${ }^{10}$ Complete pathological information is a crucial prognostic parameter to estimate the efficiency of the treatment and the probability of after-treatment relapse.

However, histopathological analysis is tedious, difficult, and time consuming as it involves gross and microscopic examination of the surgical specimen, with sample cutting and fixing, followed by preparation and examination of many slides. Moreover it is often difficult for pathologists to detect residual cancer when the response to the treatment is good but not complete. Finally, sometimes they can miss small residual cancers while making histopathological cuts. Therefore skillful and experienced pathologists are needed. Any fast and easy-to-use method able to provide relevant information on the surgical specimen such as the localization of the zones, where residual cancer may be present, or a preliminary mapping of the degree of cancer penetration can make the pathologist life easier. Subsequently this would benefit the patient.

Optical methods being fast, inexpensive, and non- or minimally invasive, have attracted considerable attention for biomedical diagnostics. ${ }^{11-14}$ Recent studies show that polarimetric imaging can provide different and supplementary information with respect to usual intensity imaging. ${ }^{15-26}$ In 
this work we present preliminary results showing that multispectral polarimetric imaging can be used after neoadjuvant treatment of colorectal cancer to detect the presence of residual tumor and evaluate the degree of cancer regression after treatment.

In this first study on this topic, we used multispectral complete Mueller imaging in order to get a complete picture of the polarimetric behavior of such samples by mapping their depolarization power, diattenuation, and retardation via suitable Mueller matrix decomposition. Simpler polarimetric measurements, such as orthogonal state contrasts, may eventually be used, but their relevance can be fully assessed only from complete Mueller studies. Moreover the spectral dependence of polarimetric images is due to the fact that hemoglobin absorption is varying with visible wavelength range, resulting in the different light penetration depth. Hence multispectral polarimetric imaging allows separation of the contributions from different layers of the sample and may be useful to optimize the optical diagnostic.

The article is organized as follows: in the next section the experimental setup and the procedure used to analyze the samples are described. The results of polarimetric imaging are presented and discussed in the third part of the paper. The last section summarizes our conclusions and outlines the perspectives of further developments.

\section{Methods}

\subsection{Experimental Setup}

The experimental setup used to analyze samples of colon ex vivo is shown in Fig. 1. A halogen lamp (a source of incoherent white light) was coupled into a fiber bundle and used to illuminate the sample. The incident light was modulated by using a polarization state generator (PSG), comprising a polarizer and two nematic liquid crystals with fixed axes and variable retardations. ${ }^{27-32}$

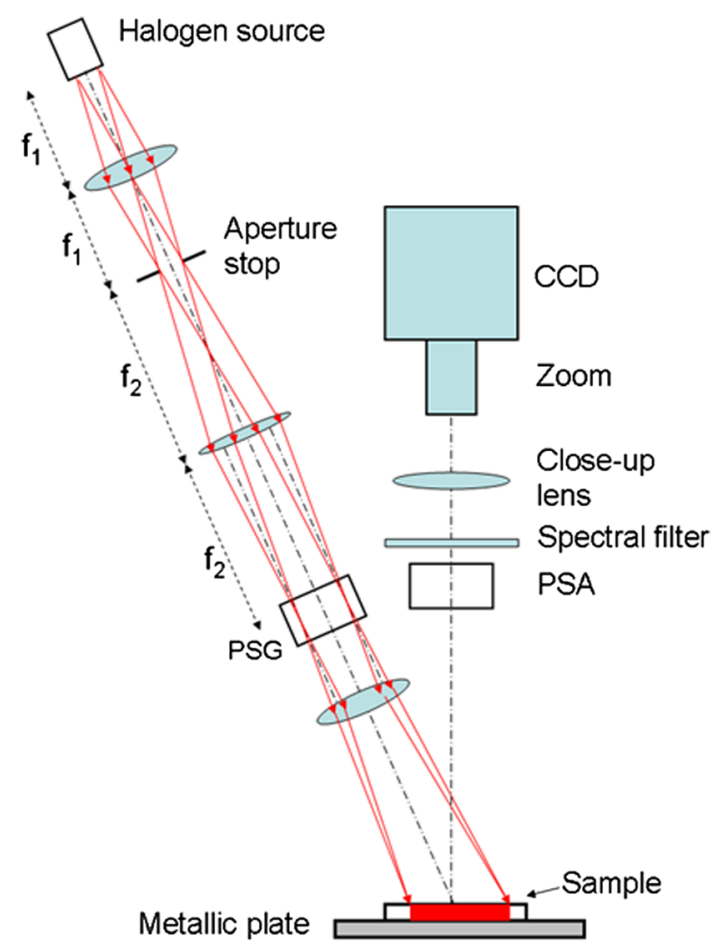

Fig. 1 Experimental setup used to analyze colon samples ex vivo.
The light backscattered by the sample was analyzed with a polarization state analyzer (PSA), a "mirror image" of the PSG, composed by the same PSG optical elements but placed in reverse order. Using a telescopic set of lenses on the illumination arm, with an iris placed at their common focus, the fiber's output is imaged inside the PSG. This experimental configuration allows us to obtain the same distribution of incidences on the surface of liquid crystals for each point of the beam. In this way the variation of the liquid crystal birefringence with the light incidence is eliminated and a spatially uniform polarization modulation over the field of view is obtained. Thereby all possible artifacts due to the limited accuracy of the positioning of the sample are also eliminated.

On the detection arm, the sample was imaged through the PSA onto a charge-coupled device camera (resolution $256 \times 256$ pixels) by means of a close-up lens (to optically "reject" the sample at infinity) followed by a zoom. By changing the zoom focal length, we could vary the size of the square field of view from 2 to $5 \mathrm{~cm}$. The wavelengths were varied from 500 to $700 \mathrm{~nm}$ in steps of $50 \mathrm{~nm}$ using $20 \mathrm{~nm}$ wide interference filters. Sixteen images, corresponding with different input and output polarization states, were acquired to measure the Mueller matrix of each sample at each wavelength. The nematic liquid crystals used to realize PSG and PSA, allowed efficient polarization modulation with the acquisition of 16 images in few seconds.

\subsection{Procedure for the Acquisition of Polarimetric Images}

The experimental setup described above was used to analyze several samples of colon ex vivo after surgery and before fixation in formalin. The excised part of colon tube was opened and placed on a cork support in order to image the mucosa, as it is the case in colonoscopy. As a result, our data should be relevant not only to pathology but also to endoscopic polarimetric imaging in the future, if this technique becomes available.

The procedure used for the analysis of each sample can be summarized in five steps:

1. The sample was placed in the position shown in Fig. 1, and a standard photo of the sample was acquired.

2. Polarimetric image was acquired without changing the position of the sample. In this way polarimetric and standard image were superimposable.

3. The zones where interesting contrasts were observed in polarimetric image were marked using different kinds of colorants.

4. The sample was blocked on a support and fixed in formalin.

5. The sampling and the analysis by pathologists of the fixed colon was realized using standard procedure. Histological cuts were realized in correspondence of the zones marked with colorant in step 3 .

\subsection{Analysis of Polarimetric Images}

As it is the case for any Mueller matrix data, the experimental polarimetric images are difficult to interpret directly. The only 
samples for which this interpretation is straightforward are the "canonical" ones, namely

- the diattenuators, or dichroic samples, for which intensity of the emerging light varies with the incident light polarization;

- the retarders, or birefringent samples, for which the phase of the emerging light varies with the incident light polarization;

- the depolarizers, which convert an incident totally polarized light into a partially polarized one. This depolarization process is typically observed when the interaction between the light and the sample is somehow "disordered," as it is the case in thick tissue sample observed in backscattering geometry; in this case the photons emerge after many scattering events on randomly distributed scatterers.

Therefore the most common procedure used to interpret a Mueller matrix is to extract its canonical properties by describing it as a product of canonical matrices, which are then determined by various decomposition methods. ${ }^{33-35}$ In this study we used the Lu-Chipman algorithm, ${ }^{34}$ which decomposes the experimentally measured Mueller matrix into a product of three "canonical" matrices: a diattenuator matrix $\mathbf{M}_{D}$ followed by a retarder matrix $\mathbf{M}_{R}$ and then followed by a depolarizer matrix $\mathbf{M}_{\Delta}$ [Eq. (1)]:

$$
\mathbf{M}=\mathbf{M}_{\Delta} \mathbf{M}_{R} \mathbf{M}_{D}
$$

The decomposition of Mueller matrices of the samples characterized in this work resulted in data reduction with both matrices $\mathbf{M}_{D}$ and $\mathbf{M}_{R}$ close to unit matrix. The values of the off-diagonal elements did not exceed the level of the measurement accuracy, which was about 2 to $3 \%$ while statistical noise was much lower than $0.5 \%$. Hence we conclude that the studied samples did not exhibit any significant diattenuation or birefringence. In the following, we show only the depolarization images (indicated with $\Delta$ ) for different wavelengths defined as:

$$
\Delta=1-\frac{1}{3}\left|\operatorname{tr}\left(\mathbf{M}_{\Delta}\right)-1\right|,
$$

where $\mathbf{M}_{\Delta}$ is the depolarization Mueller matrix. We denote the spatial average degree of depolarization by $\underline{\Delta}=\frac{1}{N} \sum_{k=1}^{N} \Delta_{k}$ where $\Delta_{k}$ indicates the degree of depolarization for each pixel of the image $\Delta$ and $N$ the number of pixels of the considered zone. Of course, once it is known that these samples are pure depolarizers, $\Delta$ can be derived directly from the measured Mueller matrices. Moreover the essential information would already be provided by linear or circular orthogonal state contrasts, which would probably be the best choice for this specific application.

\section{Results}

In this section, we first show the polarimetric response of a colorectal adenocarcinoma without any neoadjuvant treatment. Then the polarimetric images of treated colorectal cancerous samples with different responses to the treatment (cancer regression) are shown and discussed. Cancer regression was estimated by pathologists, after histological analysis, from the volume fraction of residual cancer with respect to the fibrosis replacing the original cancer.

\subsection{Colorectal Adenocarcinoma without Treatment}

The first analyzed sample (sample \#1) comes from a primitive adenocarcinoma without neoadjuvant treatment. The photo of the sample is shown in Fig. 2(a). The interface between cancerous and healthy zones [the latter being indicated by $\mathrm{H}$ in Fig. 2(a)] is observed. Two macroscopic different zones can be distinguished in cancerous part: a more external zone presenting an exophytic/budding growth with predominantly intraluminal aspect [indicated by B in Fig. 2(a)] and an inner zone presenting an endophytic/ulcerative growth with predominantly intramural aspect [indicated by $\mathrm{U}$ in Fig. 2(a)].

Polarimetric images of the sample, at different wavelengths, are shown in Fig. 2(b) to 2(d) where different zones, characterized by different degrees of depolarization of incident light, can be distinguished. These contrasts are not visible in standard photo of the sample [Fig. 2(a)]. We coded healthy and budding zones with 1 and 2, respectively [Fig. 2(b)] in polarimetric images. The degree of depolarization of ulcerated zone was observed to be spatially nonuniform: two different zones are indicated with 3 and 4 in Fig. 2(b). We observed $\underline{\Delta}(1)=\underline{\Delta}(4)>$ $\underline{\Delta}(3)>\underline{\Delta}(2)$ for all wavelengths. These contrasts decrease with increasing wavelengths. As shown below (Fig. 3), this hierarchy can be correlated with the degree of penetration of the tumor. Finally, we observed a quite general trend: for all different zones the depolarization increases with increasing wavelengths. A suitable data merging procedure might be helpful to fully take into account all the information related to the spectral dependence of the observed depolarization. Future work is planned to implement and test such a procedure. Moreover, at the bottom of Fig. 2(b) to 2(d), we observe a strongly depolarizing region (in brown), which can be identified on the photo 2(a) as pericolic tissue. This tissue, which is the outermost layer of the colon, is directly visible at the bottom of the figures as part of the transversal section of the colon wall close to the edge of the sample. Therefore we cannot take into account this part of the images. However, the strong depolarization power characteristic of the pericolic tissue accounts for the strong depolarization seen in highly ulcerated tumors at $\mathrm{T} 4$ stage, as discussed in Ref. 21.

Microscopic analysis of the sample (Fig. 3) shows that in zone 1 all layers of colon (mucosa, submucosa, muscularis propria, and subserosa) are intact. A tumoral proliferation, characterized by a strong cellular density and little concentration of tumoral stroma, is observed in zone 2 where cancerous cells spread in the submucosa (stage T1). Zone 3 is characterized by a microscopic structure similar to that of zone 2 . The ulceration on the surface decreases the cancerous wall thickness with respect to zone 2 . In zone 3 cancerous cells spread in muscular tissue (stage T2). Zone 4 is characterized by a moderate cellular density and a strong concentration of tumoral stroma. In spite of superficial ulceration, the total thickness of cancerous wall in this zone is larger than in zone 2 because abnormal cells reach the subserosa (stage T3). Mucosa, submucosa, and muscularis propria are completely destroyed in zone 4 .

Correlation between polarimetric images and histological parameters shows that different degrees of light depolarization can reveal different degrees of infiltration of cancer into the colon wall. Being T1, T2, and T3 the increasing degrees of cancer spreading in the deeper layers of colon (TNM 
(a)

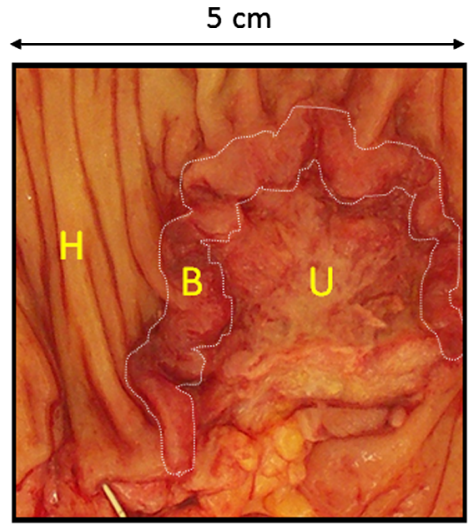

(c)

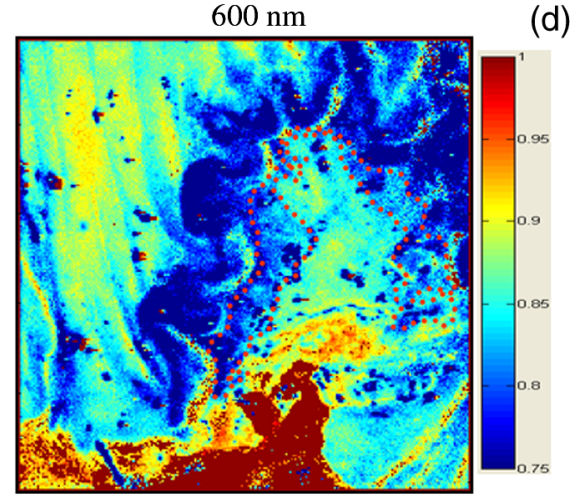

(b)

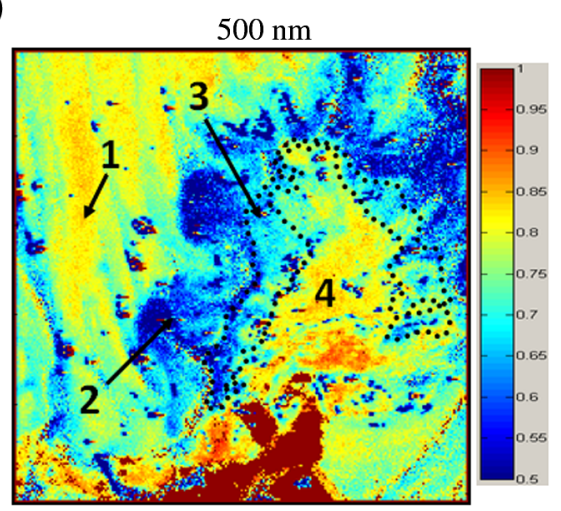

(d)

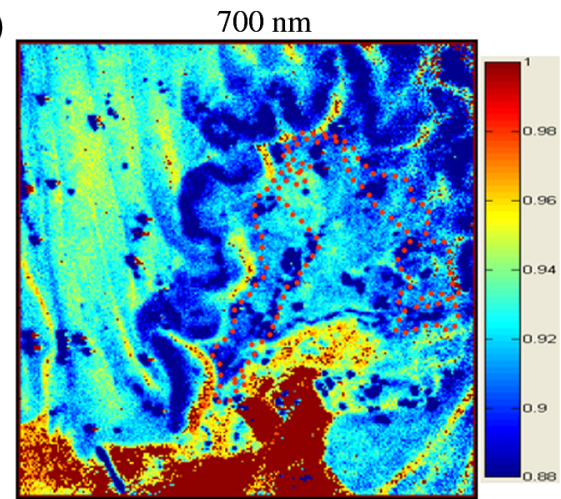

Fig. 2 (a) Photo of the first sample, a colon coming from a patient who had not been treated by radiochemotherapy. H: healthy tissue; B and U: budding and ulcerated tumoral regions, respectively. (b) to (d) Polarimetric image (total depolarization $\Delta$ ) at different wavelengths: 1 . healthy tissue; 2 . budding region; 3 and 4 . two different areas in the region ulcerated by cancer.

classification $^{8}$ ), regions at stage T3 [zone 4 in Fig. 2(b) to 2(d)] result in more depolarizing than regions at stage $\mathrm{T} 2$ [zone 3 in Fig. 2(b) and 2(d)], which result in more depolarizing than regions at stage T1 [zone 2 in Fig. 2(b) and 2(d)]. Furthermore the thickness and the microscopic structure of cancerous zone determine the specific polarimetric response of each zone. In agreement with previous investigations, ${ }^{21}$ we observed the lowest depolarization in the exophytic regions at stage T1 [zone 2 in Fig. 2(b) and 2(d)].

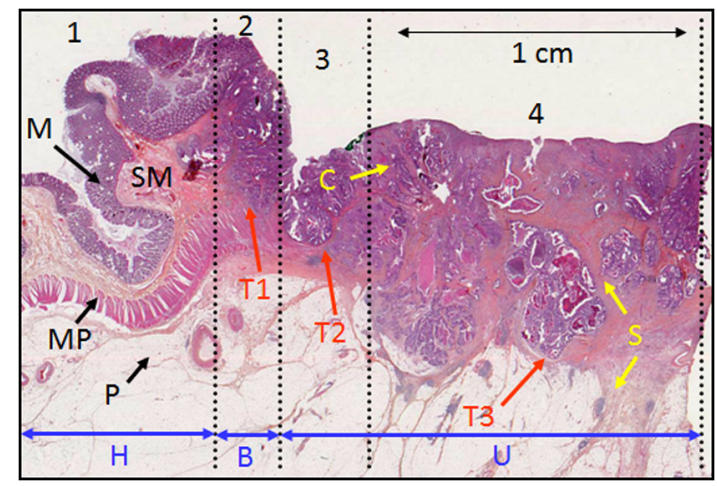

Fig. 3 Microscopic structure of the first sample: $M=$ mucosa; $\mathrm{SM}=$ submucosa; $\quad \mathrm{MP}=$ muscularis propria; $\quad P=$ pericolic tissue; $S=$ tumoral stroma; $C=$ cancerous cells. $\mathrm{H}$ is the healthy zone corresponding to zone 1 in polarimetric images; $\mathrm{B}$ is the budding zone corresponding to zone 2 in polarimetric images; $U$ is the ulcerate zone where two zones with different microscopic structures (corresponding with zones 3 and 4 in polarimetric images) can be distinguished. T1, T2, and $\mathrm{T} 3$ are the different degrees of cancer spreading in deeper layers of colon (TNM classification) for different zones.
The observed decreasing of contrasts between different zones with increasing wavelength is due to the increasing of the scattering events that the photons suffer before emerging and to the increasing of contribution by deeper layers (in particular by the strongly depolarizing pericolic tissue) to the total backscattered light for longer (more penetrating) wavelengths. This can also explain the general increasing of depolarization with increasing wavelength.

\subsection{Colorectal Adenocarcinoma after Treatment: Total Cancer Regression}

The second analyzed sample (sample \#2) was a treated colorectal adenocarcinoma featuring a total regression of cancer after treatment. Macroscopic analysis of the sample shows a reduction of the tumoral mass. In Fig. 4(a) the photo of the sample is shown. Two macroscopic different zones are observable: the healthy zone (indicated by $\mathrm{H}$ ) and the residual ulceration of the original cancer (indicated by $\mathrm{U}$ ).

Polarimetric images of the sample for different wavelengths are shown in Fig. 4(b) to 4(d), where two different zones can be distinguished: the healthy zone marked by 1 and the second zone around the ulceration marked by 2 . A contrast of $10 \%$ in the degree of depolarization between zones 1 and 2 was observed at $500 \mathrm{~nm}$ [Fig. 4(b)], zone 2 being less depolarizing than zone 1 . This contrast is no longer visible at 600 and $700 \mathrm{~nm}$ [Fig. 4(c) to 4(d)].

Microscopic analysis of the sample shows that all layers of colon wall are intact in zone 1 . In zone 2 the mucosa, muscularis mucosae, and submucosa are destroyed by the ulceration, and 
(a)

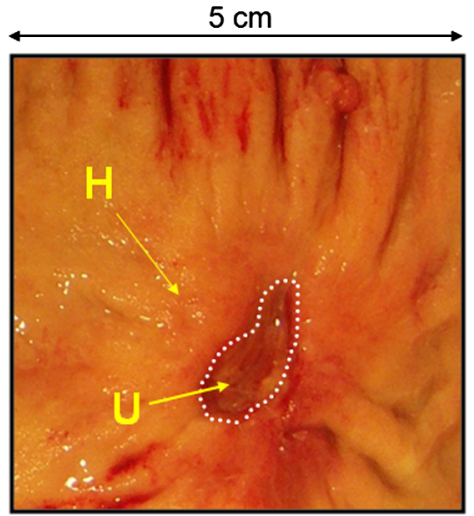

(c)

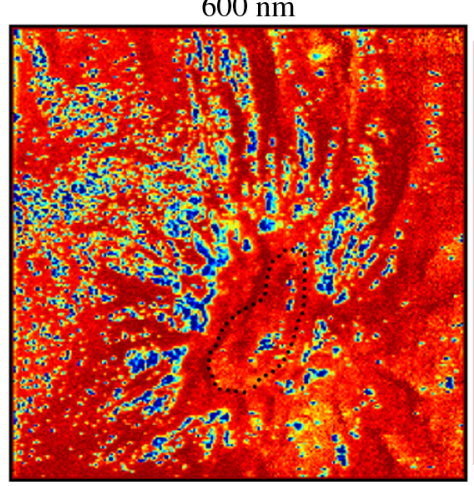

(b)

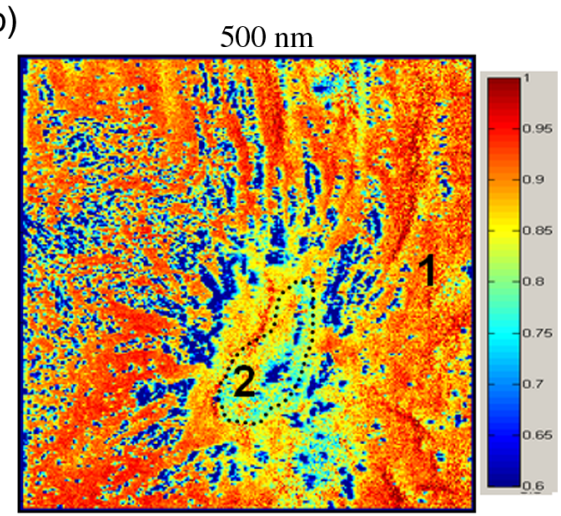

(d)

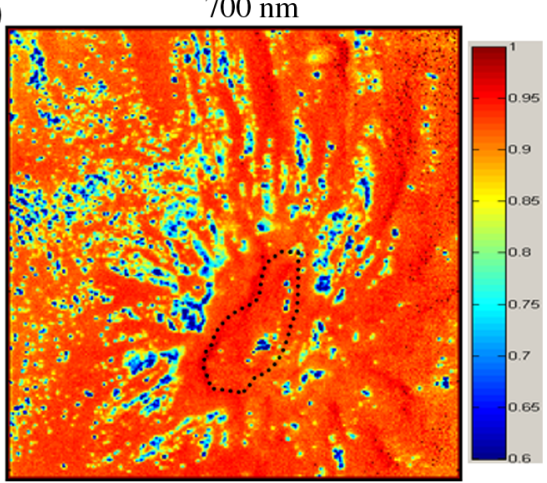

Fig. 4 (a) Photo of the second sample, a rectum resected after treating the patient by radio-chemotherapy. The "footprint" of the tumor appears as the ulcerated zone U. (b) to (d) Polarimetric images (total depolarization $\Delta$ ) at different wavelengths.

tumoral proliferation is completely removed by fibrosis: fibrous scratches spread until the fat layer [Fig. 5(a) to 5(b)].

Correlation between polarimetric images and histological parameters shows that the difference observed at $500 \mathrm{~nm}$ in the degree of depolarization between zones 1 and 2 can be attributed to the different microscopic structure of these zones and in particular to the presence of fibrosis in zone 2. Decreasing of contrast for 600 and $700 \mathrm{~nm}$ (more penetrating wavelengths) can be attributed to the increasing of the scattering events and to the contribution from deeper layers whose structures are almost the same for zones 1 and 2 .

\subsection{Colorectal Adenocarcinoma after Treatment: Good but Nontotal Cancer Regression}

The third sample (sample \#3) was a treated colorectal carcinoma where a small density of residual cancer was observed.
Macroscopic analysis of the sample showed a macroscopic reduction of tumoral mass. The photo of the sample is shown in Fig. 6(a). Healthy zone and residual ulceration of the original cancer are indicated by $\mathrm{H}$ and $\mathrm{U}$, respectively. We did not consider for the analysis the zone $\mathrm{N}$, which corresponds with the transverse section of the colon wall.

In polarimetric images [Fig. 6(b) to 6(d)], three principal zones with different degrees of depolarization can be distinguished [Fig. 6(b)]: zone 1 corresponds with the healthy zone, whereas zones 2 and 3 correspond with two different areas in ulcerated zone. For $500 \mathrm{~nm}$, we observed $\underline{\Delta}(1)>\underline{\Delta}(2)>$ $\Delta(3)$. Again, the contrasts between selected zones change for 600 and $700 \mathrm{~nm}$, with an overall increase of depolarization with increasing wavelengths.

Microscopic analysis of this sample (Fig. 7) shows that in zones 2 and 3 a strong ulceration of the surface is present, (a)

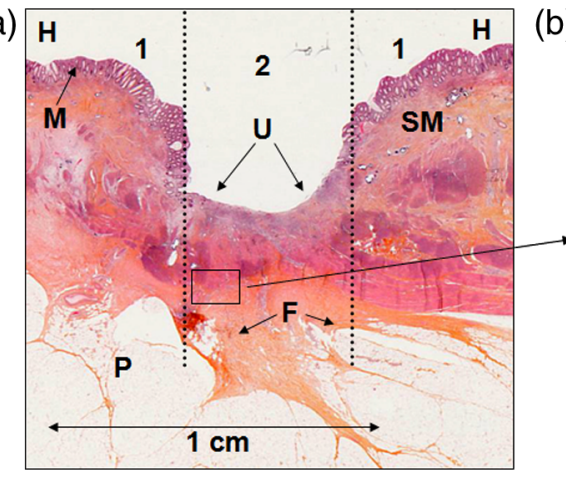

(b)

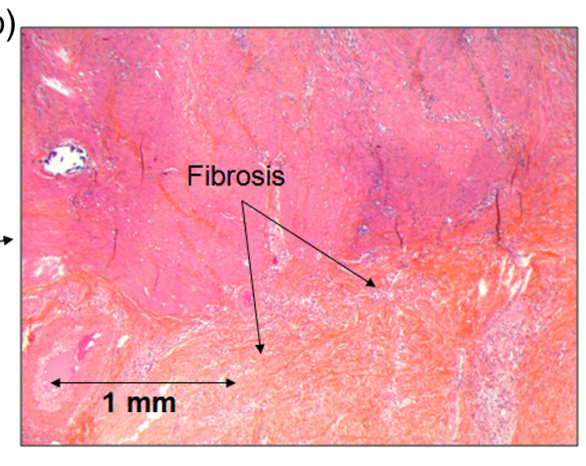

Fig. 5 Microscopic structure of the second sample: $M=$ mucosa; $\mathrm{SM}=$ submucosa; $P=$ pericolic tissue; $F=$ fibrosis. $H$ is the healthy zone corresponding with zone 1 in polarimetric images; $U$ is the ulcerate zone corresponding with zone 2 in polarimetric images. 
(a)

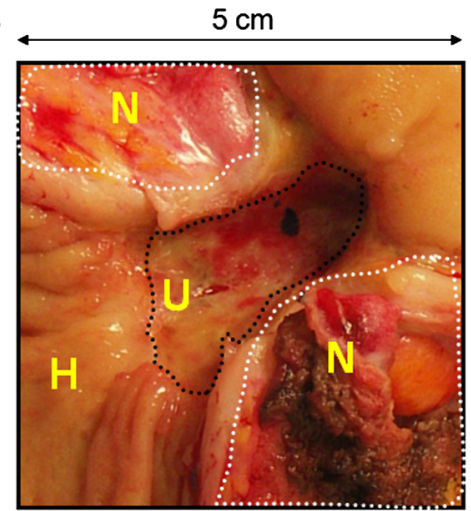

(c)

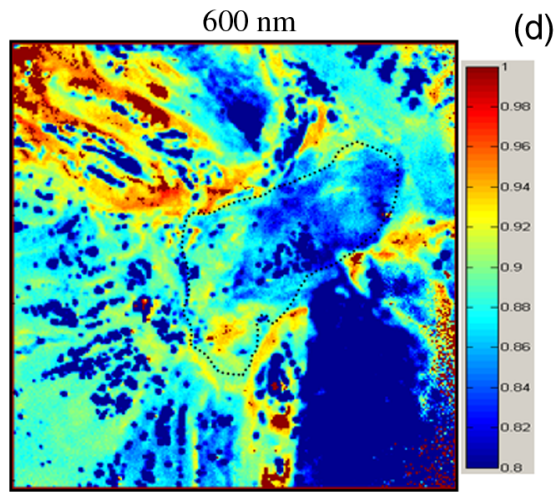

(b)

(d)
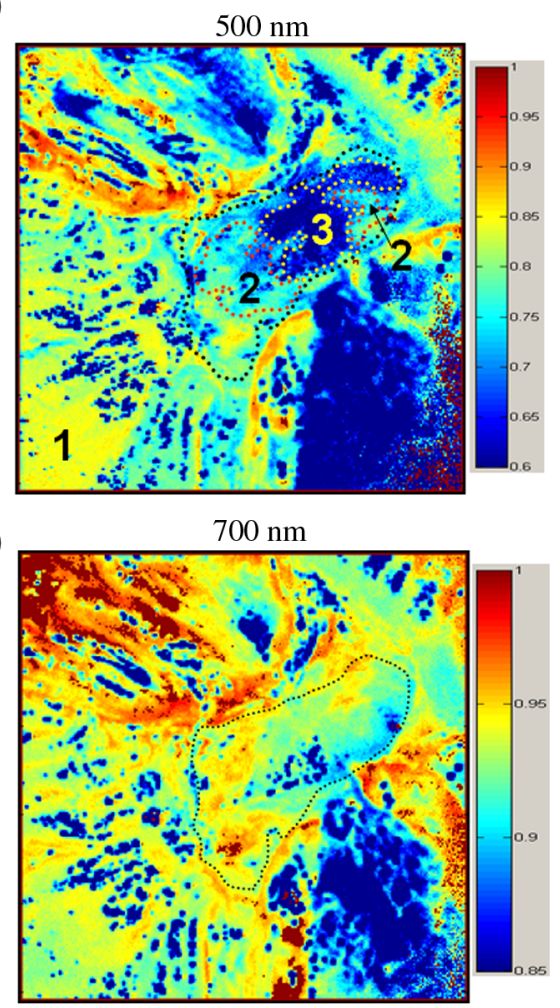

Fig. 6 (a) Photo of the third sample, a rectum resected after treating the patient by radio-chemotherapy. The footprint of the tumor appears as the ulcerated zone U. (b) to (d) Polarimetric image (total depolarization $\Delta$ ) at different wavelengths.

and fibrosis replaces the original cancer. In addition, in zone 3 a residual cancer, with a volume fraction equal to $5 \%$ of the original cancer, was observed. This figure denotes a good but nontotal response of the tumor to the treatment. Fibrosis is estimated to occupy $95 \%$ of original cancer volume. Tumoral stroma is present in the zones surrounding abnormal cells.

Correlation between polarimetric images and histological parameters shows that the fibrosis accounts for the $15 \%$ difference in the depolarization between zones 2 and 1 (as in the sample \#2) while the presence of residual cancer accounts for the $30 \%$ difference in the depolarization powers between the zones 3 and 1 at $500 \mathrm{~nm}$. Also for this sample decreasing of contrast for 600 and $700 \mathrm{~nm}$ (more penetrating wavelengths) can be attributed to the increasing of the scattering events and to the contribution from deeper layers untouched by cancer and in particular to the strongly depolarizing pericolic tissue.

\subsection{Colorectal Adenocarcinoma after Treatment: Little Cancer Regression}

The fourth and last sample (sample \#4) was a treated colorectal adenocarcinoma with a significant density of residual cancerous cells. The photo is shown in Fig. 8(a) where two macroscopic (a)

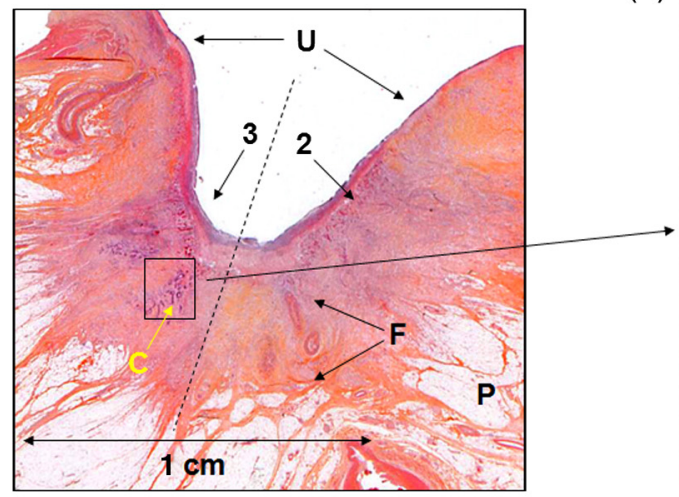

(b)

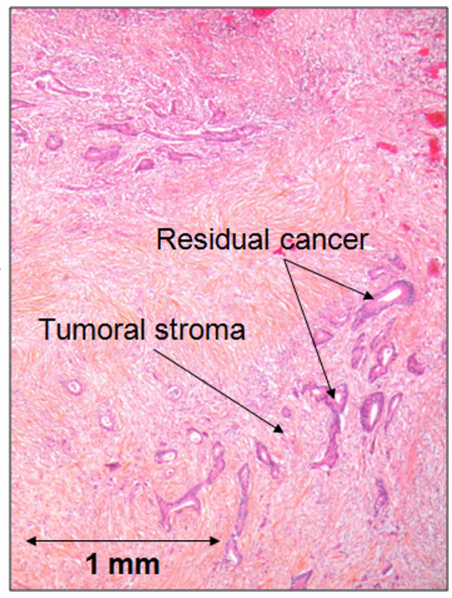

Fig. 7 Microscopic structure of the second type of analyzed sample after radio-chemotherapy treatment: $P=$ pericolic tissue; $F=$ fibrosis; $C=$ residual cancer after treatment. $U$ is the ulcerate zone corresponding with zone 2 and 3 in polarimetric images. 
(a)

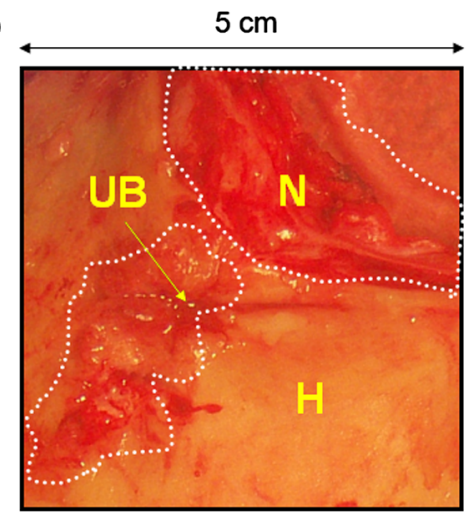

(c)

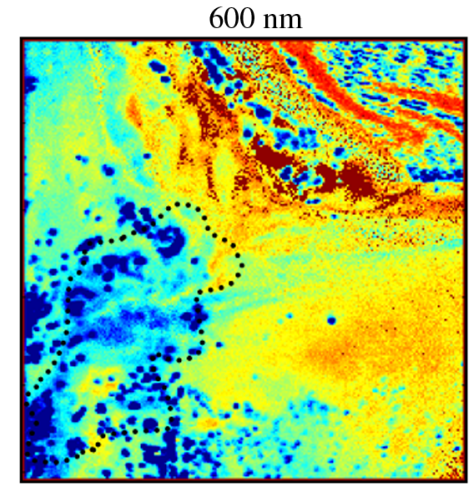

(b)

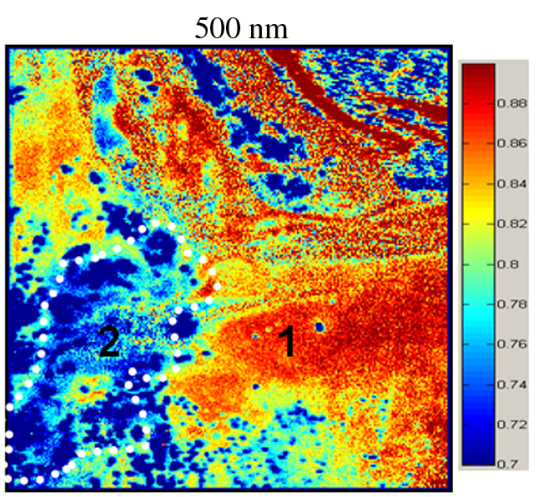

(d)

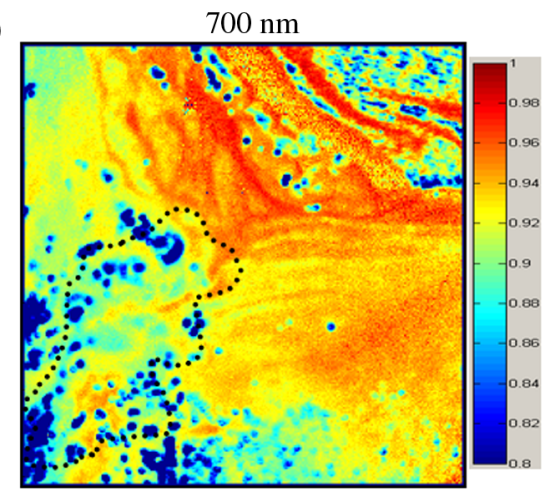

Fig. 8 (a) Photo of the fourth sample, a rectum resected after treating the patient by radio-chemotherapy. (b) to (d) Polarimetric images (total depolarization $\Delta$ ) at different wavelengths.

zones can be distinguished: the healthy zone indicated by $\mathrm{H}$ and the zone indicated by UB, where both ulceration and small budding zones are present. We did not consider for the analysis the zone $\mathrm{N}$, which corresponds with the transverse section of the colon wall. In polarimetric images [Fig. 8(b) to 8(d)], we can distinguish two zones with different degrees of depolarization: zones 1 and 2, respectively, corresponding with the healthy tissue and the area of the ulcero-budding zone. For $500 \mathrm{~nm}$, we found $\underline{\Delta}(1)>\underline{\Delta}(2)$. Also in this case contrast changes with increasing wavelength, with the same overall trends as previous analyzed samples.

Microscopic analysis (Fig. 9) shows that for zone 1 all layers of colon are intact. In zone 2 a residual cancer after treatment is present. In this case a little tumoral regression after treatment is observed. The residual cancer, characterized by a strong cellular density and a non-negligible percentage of tumoral stroma (microscopic structure similar to the zone 4 for the first nontreated sample) is estimated to occupy $70 \%$ of the initial volume of the cancer.

Correlation between polarimetric images and histological parameters shows that the different degree of depolarization between zones 1 and 2 can be explained by high density of residual cancerous cells, non-negligible presence of tumoral stroma after treatment, and small percentage of fibrotic areas (observed principally in the pericolic tissue) in the zone 2 .

The reason for the lower contrast at 600 and $700 \mathrm{~nm}$ is the same as discussed earlier for the above two samples (sample \#2 and sample \#3).
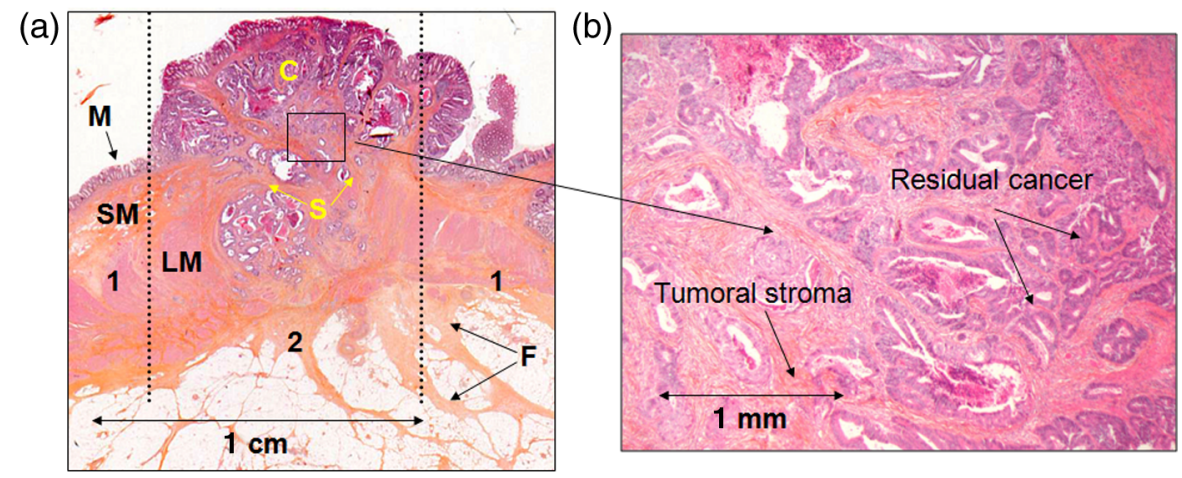

Fig. 9 Microscopic structure of the fourth sample: $M=$ mucosa; $S M=$ submucosa; $L M=$ longitudinal muscular tissue; $F=$ fibrosis; $C=$ residual cancerous cells after treatment; $S=$ tumoral stroma. Zone 2 corresponds with ulcero-budding zone (UB) in the photo of the sample; zone 1 corresponds with healthy zone $(\mathrm{H})$ in the photo of the sample. 


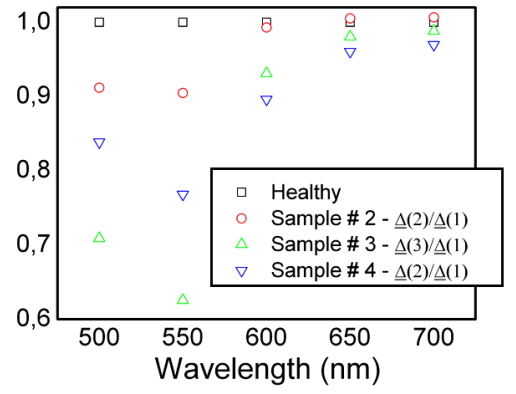

Fig. 10 Polarimetric response for different degrees of cancer regression after neoadjuvant treatment.

\section{Discussion}

\subsection{Colorectal Carcinomas after Treatment: Summary}

Finally, to compare the experimental results for different treated samples, we normalized the average depolarization of the zones corresponding to the fibrosis and/or residual cancer (zone 2 for samples \#2 and \#4, zone 3 for sample \#3) by the average degree of depolarization of the healthy zone (named zone 1 for all analyzed samples) of the same sample. The results are shown in Fig. 10.

At 500 to $550 \mathrm{~nm}$, the normalized total depolarization does not vary monotonically with the degree of cancer regression. This effect is likely to be related to the lower depth of penetration of the green light that increases sensitivity of measurements to features of the most superficial layers. For these wavelengths, polarized light is more sensitive to microscopic differences among different analyzed samples. This trend is due to different densities of abnormal cells and tumoral stroma with respect to the fibrosis and to the different morphological properties of tumoral stroma of each sample also witnessed by different responses to the treatment. Biochemical and morphological properties of stroma and the interactions between abnormal cells and extracellular matrix are crucial for cancer development $^{36}$ and for the response of patient to the treatment. At 600,650 , and $700 \mathrm{~nm}$, the contrasts vary monotonically with the degree of cancer regression, which is of course an interesting trend for diagnostics. On the other hand, for larger wavelengths all tissues become more depolarizing due to larger penetration depths, leading to smaller absolute contrasts in depolarization images than those observed in the green part of the spectrum. It is therefore difficult at this point to make a definitive choice of the optimal wavelength for residual cancer diagnostic.

Suitable combinations of images taken at various wavelengths are more likely to eventually optimize the diagnostic performance. Of course, for this to be done efficiently it would be necessary to better identify the histological parameters affecting the polarimetric response of the tissue. Surely the microscopic structure (cellular density, stroma, blood concentration, fibrosis) and thickness of cancerous wall influence the penetration of light and the polarimetric response for different wavelengths for both nontreated and treated (with different degrees of cancer regression) colorectal adenocarcinomas. The relative weights of these contributions will be investigated in more detail in future work on larger sample collections.

\section{Conclusion}

In this work we show that polarimetric imaging exhibits interesting contrasts between the footprints of cancerous parts and surrounding tissue on rectum samples taken on patients after neaodjuvant treatment. The general trends are similar to those seen on untreated colon samples: these samples are pure depolarizers, with depolarization increasing with increasing wavelengths. When a residual tumor is present, the footprints of initial tumoral zones are less depolarizing than the surrounding healthy tissues, this contrast being sensitive enough to allow easy detection of residual tumors with volume fractions as small as a few percent.

These results, though still preliminary, show that multispectral polarimetric imaging is a promising tool to detect residual cancer and provide a first evaluation of cancer regression in colorectal carcinomas after neoadjuvant treatment.

In the future this technique might also be used in vivo, if polarimetric endoscopy becomes available to guide biopsies and help pathologists in the determination/improvement of prognostic and predictive markers to orient presurgery strategy.

\section{Acknowledgments}

This work was funded in part by the Institut National du Cancer (INCa) and the Cancéropôle, under contract \#2012-1-GYN-01EP-1.

\section{References}

1. International Agency for Research on Cancer, "Globocan 2008, Estimated cancer Incidence, Mortality, Prevalence and Disabilityadjusted life years (DALYs) Worldwide in 2008," http://globocan.iarc fr/.

2. G. J. Frykholm, L. Pahlman, and B. Glimelius, "Combined chemo- and radiotherapy vs. radiotherapy alone in the treatment of primary, nonresectable adenocarcinoma of the rectum," Int. J. Radiat. Oncol. Biol. Phys. 50(2), 427-434 (2001).

3. J. F. Bosset et al., "Chemotherapy with preoperative radiotherapy in rectal cancer," N. Engl. J. Med. 355(11), 1114-1123 (2006).

4. J. P. Gérard et al., "Preoperative radiotherapy with or without concurrent fluorouracil and leucovorin in T3-T4 rectal cancers: results of FFCD 9203," J. Clin. Oncol. 24(28), 4620-4625 (2006).

5. M. Braendengen et al., "Randomized phase III study comparing preoperative radiotherapy with chemotherapy in non-resectable rectal cancer," J. Clin. Oncol. 26(22), 3687-3694 (2008).

6. O. Visser et al., "The influence of total mesorectal excision on local recurrence and survival in rectal cancer patients: a population-based study in Greater Amsterdam," J. Surg. Oncol. 95(6), 447-454 (2007).

7. P. Quirke et al., "Local recurrence of rectal adenocarcinoma due to inadequate surgical resection. Histopathological study of lateral tumour spread and surgical excision," Lancet 2(8514), 996-999 (1986).

8. L. H. Sobin, M. K. Gospodarowicz, and C. Wittekind, Eds., TNM Classification of Malignant Tumors, 7th ed., Wiley-Blackwell, Oxford (2009).

9. A. Wibe et al., "Prognostic significance of the circumferential resection margin following total mesorectal excision for rectal cancer," $\mathrm{Br}$. J. Surg. 89(3), 327-334 (2002).

10. J. Shia et al., "Patterns of morphologic alteration in residual rectal carcinoma following preoperative chemoradiation and their association with long-term outcome," Am. J. Surg. Pathol. 28(2), 215-223 (2004).

11. D. Huang et al., "Optical coherence tomography," Science 254(5035), 1178-1181 (1991).

12. W. Denk, J. H. Strickler, and W. W. Webb, "2-photon laser scanning fluorescence microscopy," Science 248(4951), 73-76 (1990).

13. Y. Guo et al., "Second-harmonic tomography of tissues," Opt. Lett. 22(17), 1323-1325 (1997).

14. J. B. Pawley, Handbook of Biological Confocal Microscopy, Springer, New York (2006).

15. S. L. Jacques et al., "Polarized light camera to guide surgical excision of skin cancers," Proc. SPIE 6842, 68420I (2008). 
16. M. F. G. Wood et al., "Proof-of-principle demonstration of a Mueller matrix decomposition method for polarized light tissue characterization in vivo," J. Biomed. Opt. 14(1), 014029 (2009).

17. S. G. Demos, H. B. Radousky, and R. R. Alfano, "Deep surface imaging in tissue using spectral and polarization filtering," Opt. Express 7(1), 23-28 (2000).

18. A. H. Hielscher, J. R. Mourant, and I. J. Bigio, "Influence of particle size and concentration on the diffuse backscattering of polarized light from tissue phantoms and biological cell suspensions," Appl. Opt. 36(1), 125-135 (1997).

19. M. R. Antonelli et al., "Mueller matrix imaging of human colon tissue for cancer diagnostics: how Monte Carlo modelling can help in the interpretation of experimental data," Opt. Express 18(10), 1020010208 (2010).

20. M. H. Smith et al., "Mueller matrix imaging polarimetry in dermatology," Proc. SPIE 3911, 210-216 (2000).

21. A. Pierangelo et al., "EX-vivo characterization of human colon cancer by Mueller polarimetric imaging," Opt. Express 19(2), 1582-1593 (2011).

22. A. Pierangelo et al., "Use of Mueller imaging for the staging of human colon cancer," Proc. SPIE 7895, 78950E (2011).

23. M. R. Antonelli et al., "Impact of model parameters on Monte Carlo simulations of backscattering Mueller matrix images of colon tissue," Biomed. Opt. Express 2(7), 1836-1851 (2011).

24. O. Aharon et al., "Differential optical spectropolarimetric imaging system assisted by liquid crystal devices for skin imaging," J. Biomed. Opt. 16(8), 086008 (2011).

25. I. Abdulhalim, R. Moses, and R. Sharon, "Biomedical optical applications of liquid crystal devices," Acta Physica Polonica A 112(5), 715-722 (2007)
26. A. Safrani et al., "Skin biomedical optical imaging system using dualwavelength polarimetric control with liquid crystals," J. Biomed. Opt. 15(2), 026024 (2010).

27. A. De Martino et al., "Optimized Mueller polarimeter with liquid crystals," Opt. Lett. 28(8), 616-618 (2003).

28. B. Laude-Boulesteix et al., "Mueller polarimetric imaging system with liquid crystals," Appl. Opt. 43(14), 2824-2832 (2004).

29. E. Garcia-Caurel, A. DeMartino, and B. Drévillon, "Spectroscopic Mueller polarimeter based on liquid crystal devices," Thin Solid Films 455-456, 120-123 (2004).

30. A. De Martino et al., "General methods for optimized design, and calibration of Mueller polarimeters," Thin Solid Films 455-456, 112-119 (2004).

31. E. Compain, S. Poirier, and B. Drevillon, "General and self-consistent method for the calibration of polarization modulators, polarimeters, and Mueller-matrix ellipsometers," Appl. Opt. 38(16), 3490-3502 (1999).

32. O. Aharon and I. Abdulhalim, "Liquid crystal wavelength independent continuous polarization rotator," Opt. Eng. 49(3), 034002 (2010).

33. R. Ossikovski, A. De Martino, and S. Guyot, "Forward and reverse product decompositions of depolarizing Mueller matrices," Opt. Lett. 32(6), 689-691 (2007).

34. S.-Y. Lu and R. A. Chipman, "Interpretation of Mueller matrices based on polar decomposition," J. Opt. Soc. Am. A 13(5), 1106-1113 (1996).

35. C. Fallet et al., "Experimental validation of the symmetric decomposition of Mueller matrices," Opt. Express 18(2), 831-842 (2010).

36. T. D. Tlsty and L. M. Coussens, "Tumor stroma and regulation of cancer development," Аппи. Rev. Pathol. 1, 119-150 (2006). 